\title{
Erratum: Eating speed and the risk of type 2 diabetes: explorations based on real-world evidence
}

Sai Krishna Gudi, BPharm, PharmD, MSc

College of Pharmacy, Rady Faculty of Health Sciences, University of Manitoba, Winnipeg, MB, Canada https://doi.org/10.6065/apem.2040028.014

Ann Pediatr Endocrinol Metab 2020;25(2):80-83.

In this article, the author's PhD degree was written unintentionally. The PhD degree should be deleted as follows:

\section{Corrected the author's degree}

Sai Krishna Gudi, BPharm, PharmD, MSc 